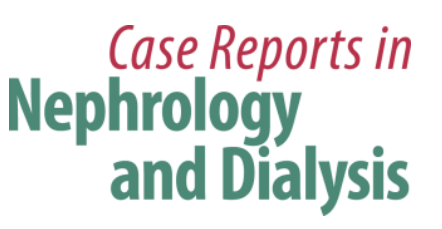

Case Rep Nephrol Dial 2019;9:49-54

DOI: 10.1159/000500296

Published online: May 16, 2019

(c) 2019 The Author(s)

Published by S. Karger AG, Basel

www.karger.com/cnd

This article is licensed under the Creative Commons Attribution-NonCommercial 4.0 International License (CC BY-NC) (http://www.karger.com/Services/OpenAccessLicense). Usage and distribution for commercial purposes requires written permission.

\title{
IgG4-Related Kidney Disease: A Curious Case of Interstitial Nephritis with Hypocomplementemia
}

\author{
Emmett Tsz Yeung Wong ${ }^{a}$ Manjari Lahiri ${ }^{b}$ Ming Teh $^{c}$ \\ Christopher Cheang Han Leo ${ }^{a}$ \\ aDivision of Nephrology, University Medicine Cluster, National University Hospital, \\ Singapore, Singapore; ${ }^{b}$ Division of Rheumatology, University Medicine Cluster, \\ National University Hospital, Singapore, Singapore; 'Department of Pathology, \\ National University Hospital, Singapore, Singapore
}

\section{Keywords}

Tubulointerstitial disease - IgG4-related kidney disease - Acute kidney injury . Hypocomplementemia $\cdot$ Acute interstitial nephritis

\begin{abstract}
IgG4-related kidney disease has been relatively newly recognized over the last two decades as a combination of an autoimmune and allergic disorder, with elevated serum IgG4 level and hypocomplementemia among its characteristic features. Here we report the case of a man with interstitial nephritis presenting with acute kidney injury and hypocomplementemia but normal serum IgG4 level and provide a literature review of IgG4-related kidney disease. This case highlights the importance of IgG4-related kidney disease as an important differential diagnosis in any patient presenting with a clinical syndrome mimicking acute interstitial nephritis with hypocomplementemia. A high index of suspicion with a low threshold for performing a native kidney biopsy would be paramount as patients do respond well to corticosteroid therapy.
\end{abstract}




\section{Case Reports in Nephrology and Dialysis}

Wong et al.: IgG4-Related Kidney Disease: A Curious Case of Interstitial Nephritis with Hypocomplementemia

\section{Introduction}

IgG4-related kidney disease (IgG4-RKD) has been relatively newly recognized over the last two decades as a combination of an autoimmune and allergic disorder, with elevated serum IgG4 level and hypocomplementemia among its characteristic features. We present a case of a man with interstitial nephritis presenting with acute kidney injury, lower urinary tract symptoms, and loss of weight, with hypocomplementemia but normal serum IgG4 levels.

\section{Case Report/Case Presentation}

A 66-year-old man of Indian ethnicity presented to our institution for management of acute kidney injury. He first presented to another hospital (A) 4 days prior with lower urinary tract symptoms, upper respiratory tract symptoms, and loss of weight and was found to have acute kidney injury (creatinine $561 \mu \mathrm{mol} / \mathrm{L}$; last known creatinine of $60 \mu \mathrm{mol} / \mathrm{L}$ a year ago) with hyperkalemia and metabolic acidosis.

The next day, he was transferred to another hospital (B) for a second opinion. In addition to intravenous fluids, he was started on intravenous ceftriaxone. Further investigations were performed to determine the etiology of kidney impairment. Urine protein on dipstick was 1+. Of note, anti-nuclear antibody titer was elevated at 1:160 (homogenous pattern by immunofluorescence), and there was hypocomplementemia (C3 $0.32 \mathrm{~g} / \mathrm{L}$; ref.: 0.90-1.80 g/L; C4 $<0.060 \mathrm{~g} / \mathrm{L}$; ref.: $0.10-0.40 \mathrm{~g} / \mathrm{L}$ ). Antibodies against proteinase 3 (anti-PR3) and myeloperoxidase (anti-MPO) were within normal range, and antibodies against double-stranded DNA (anti-dsDNA) were negative. Computed tomography of the kidneys, ureters, and urinary bladder revealed edematous kidneys with bilateral perinephric stranding and faint densities in the collecting system of both kidneys, but no evidence of hydronephrosis, ureteric stones, or obstructive uropathy. There was moderate prostatomegaly.

He was subsequently transferred to our institution for further management of kidney impairment and for consideration of a kidney biopsy.

He has a 20 -year history of type 2 diabetes mellitus (HbA1c 6.8\%) with bilateral moderate non-proliferative diabetic retinopathy and maculopathy. His medical history also includes hyperlipidemia, right cubital tunnel syndrome, a history of hemorrhagic renal cyst, gastritis, duodenitis, and a bulky left adrenal gland.

Further history revealed that he had noticed frothy urine with anorexia and weight loss of $5 \mathrm{~kg}$ over the past 3 months. A review of systems, in particular for rash, arthralgias, myalgias, sicca, and Raynaud's phenomena, was unremarkable. His medication history revealed that he had consumed traditional Chinese medications, co-trimoxazole and non-steroidal antiinflammatory drugs (NSAIDs) in the past month. There was no family history of rheumatic diseases or malignancies. The physical examination was unremarkable.

Initial urinalysis showed mild pyuria (WBC 3/high-power field) and microscopic hematuria (RBC 103/high-power field), with a spot urinary protein-to-creatinine ratio of 163 $\mathrm{mg} / \mathrm{mmoL}$ (estimated total urinary protein of $1.4 \mathrm{~g} /$ day). Screening laboratory tests for viral hepatitis, HIV, and cryoglobulins were negative. There was persistent hypocomplementemia (C3 39 mg/dL; ref.: 85-185 mg/dL; C4 <8 mg/dL; ref.: 10-50 mg/dL).

Subsequently, a native kidney biopsy was performed. The histopathology reports the following: two out of fifteen glomeruli are globally sclerotic, with the rest of the glomeruli within normal limits with no increase in mesangial matrix or cellularity. No endocapillary proliferation or crescent is identified. Glomerular basement membranes appear unremarkable on 
silver stains. There is approximately $60-70 \%$ tubular atrophy or partial tubular atrophy. There is prominent interstitial edema and extensive inflammation composed predominantly of mononuclear or lymphoplasmacytic cells admixed with scattered neutrophils and eosinophils. No granulomas are identified. There is mild hyaline arteriolosclerosis. Immunofluorescence pattern was negative.

Based on the histology, a provisional diagnosis of acute interstitial nephritis with severe interstitial fibrosis and tubular atrophy (IFTA) was made, with co-trimoxazole or NSAIDs being possible drug culprits.

However, in view of the severe hypocomplementemia, a differential diagnosis of IgG4related tubulointerstitial nephritis was considered. Corticosteroid therapy (prednisolone $1 \mathrm{mg} / \mathrm{kg} /$ day) was started for the acute tubulointerstitial nephritis with the underlying differential diagnosis being IgG4-related disease, tubulointerstitial nephritis with uveitis (TINU), systemic lupus erythematosus, or Sjögren's disease.

Special stains for IgG4 show more than 30 IgG4-positive plasma cells per high-power field (Fig. 1a, b). Fibrosis appears to have a storiform pattern on trichrome stains (Fig. 1c) and the inflammation has a zonal character with one core showing relative un-involvement at one end (Fig. 1d, e). Of note, there was no obliterative phlebitis.

Serum IgG subclasses returned with a raised total IgG (2,740 mg/dL; ref.: 767-1,590 mg/dL), IgG1 (1,920 mg/dL; ref.: 341-894 mg/dL), IgG2 (957 mg/dL; ref.: 171-632 mg/dL), and IgG3 (334.0 mg/dL; ref.: $18.4-106.0 \mathrm{mg} / \mathrm{dL}$ ), but normal IgG4 (44.0 mg/dL; ref.: 2.4-121.0 $\mathrm{mg} / \mathrm{dL}$ ). No antibodies to anti-extractable nucleic acid (ENA) antibodies were detected.

Electron microscopy of kidney biopsy tissue was eventually reported, showing prominent flattening of podocytes without evidence of electron dense deposits.

A positron emission tomography (PET) revealed mildly FDG-avid small volume lymphadenopathy above and below the diaphragm, heterogeneous cortical increased FDG uptake within both kidneys, and patchy mildly increased FDG uptake along bilateral upper ribs.

Two weeks after treatment with corticosteroid therapy, there was improvement in his kidney function (Fig. 2), microscopic hematuria, and proteinuria (estimated total urinary protein of $0.2 \mathrm{~g} /$ day, 2 weeks after treatment).

\section{Discussion/Conclusion}

IgG4-related disease (IgG4-RD) has been likened to "a black crow flying through the dark night" as it passed through the history of medicine, until the concept of an overarching diagnosis unified conditions once regarded as isolated, single-organ diseases [1, 2]. Elevated IgG4 levels were first linked to autoimmune pancreatitis in 2001 [3] and were subsequently recognized as a systemic condition in 2003 after extrapancreatic manifestations were identified [4].

IgG4-RKD occurs in 15\% of patients with IgG4-RD [5] and include a wide range of manifestations such as tubulointerstitial nephritis, membranous glomerulonephropathy, pyelitis, and hydronephrosis due to retroperitoneal fibrosis [6].

Two major clinical presentations are recognized - unexplained renal dysfunction and imaging abnormalities. Elevated serum IgG4 level and hypocomplementemia are characteristic features of IgG4-RKD [7, 8]. The key morphologic features of IgG4-RD are a dense lymphoplasmacytic infiltrate that is organized in a storiform pattern, obliterative phlebitis, and a mild-to-moderate eosinophil infiltrate [8]; usually, more than 10 infiltrating IgG4 and plasma cells per high-power field or at least $40 \%$ of the ratio of IgG4 and plasma cells to IgG and plasma cells are employed as the cutoff values [7]. 


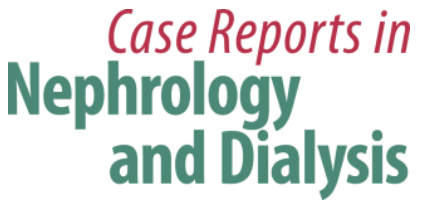

Case Rep Nephrol Dial 2019;9:49-54

DOI: $10.1159 / 000500296$

(c) 2019 The Author(s). Published by S. Karger AG, Basel www.karger.com/cnd

Wong et al.: IgG4-Related Kidney Disease: A Curious Case of Interstitial Nephritis with Hypocomplementemia

The patient described in this case report had tubulointerstitial nephritis with a storiform pattern of fibrosis on histology of his kidney biopsy, but without findings of obliterative phlebitis. Interestingly, all subclasses of IgG were elevated with the exception of IgG4. There had been case reports of IgG4-negative IgG4-RD in which patients showed typical clinical, imaging, and histopathological features of IgG4-RD, despite normal serum IgG4 levels and very few IgG4 and plasma cell infiltrates in the affected organs [7, 9, 10]. As such, an elevated serum IgG4 level is strongly supportive, but not a mandatory diagnostic criterion for diagnosis of IgG4-RKD. This is especially so in patients with significant proteinuria, whose serum IgG and IgG4 levels may both be reduced. Compatible histological features supportive of IgG4-RKD are by themselves adequate for diagnosis, irrespective of serum IgG4 levels.

There has also been a study on the prozone phenomenon, a false negative response resulting from high antibody titer which interferes with formation of antigen-antibody, resulting in spuriously low serum IgG4 concentrations in patients with IgG4-RD [11]. Whether IgG4 negativity within such a clinical context suggests a condition that mimics IgG4-RD or is attributable to the prozone phenomenon remains an area for future research. In the case of our patient, the plasma was not diluted prior to measurement of IgG4 levels, and serial IgG4 levels were also not measured in subsequent clinic reviews. At present, there is inadequate evidence for monitoring of serum IgG4 levels as a marker of disease activity, especially in cases where serum IgG4 levels are not elevated to begin with.

A recent study by Wang et al. [12] of a Chinese cohort of patients with biopsy-proven IgG4-RKD also illustrated the rarity of the disease in their study population with only a $0.042 \%$ incidence of the disease from 40,429 native kidney biopsy cases from January 2010 to January 2018. They also found that patients with a low C3 had poorer prognosis, likely due to more severe renal fibrosis found in this group in comparison to those with a normal C3 level. This could be pertinent to our patient's expected prognosis as his C3 levels were markedly reduced at presentation as well. The complement levels returned to normal in our patient after 3 months of corticosteroid therapy with a corresponding improvement of the renal function. This certainly brings to question the role complements have in the pathophysiology of IgG4RKD.

In conclusion, this case report highlights the possibility for the existence of IgG4-RKD even with normal serum IgG4 levels. Despite being a rare disease, IgG4-RKD remains an important differential diagnosis in any patient presenting with a clinical syndrome mimicking acute interstitial nephritis with hypocomplementemia. A high index of suspicion with a low threshold for performing a native kidney biopsy would be paramount in making the appropriate diagnosis (with IgG4 staining) in this case as they do respond well to corticosteroid therapy. A more universal use of IgG4 staining in all kidney biopsies with tubulointerstitial nephritis should be strongly considered given this new and interesting phenomenon.

\section{Statement of Ethics}

The authors have no ethical conflicts to disclose. Written informed consent was obtained from the patient for publication of this case report, including the histology images.

\section{Disclosure Statement}

The authors have no conflicts of interest to declare. 


\section{Author Contributions}

E.T.Y.W. contributed to acquisition of data and writing of the manuscript. M.T. contributed to preparation and annotation of the histology. M.L. and C.C.H.L provided supervision and mentorship.

\section{References}

1 Saito T, Stone JH, Nakashima H, Saeki T, Kawano M. IgG4-related kidney disease. Japan: Springer; 2016. https://doi.org/10.1007/978-4-431-55687-9.

2 Kamisawa T, Zen Y, Pillai S, Stone JH. IgG4-related disease. Lancet. 2015 Apr;385(9976):1460-71.

3 Hamano H, Kawa S, Horiuchi A, Unno H, Furuya N, Akamatsu T, et al. High serum IgG4 concentrations in patients with sclerosing pancreatitis. N Engl J Med. 2001 Mar;344(10):732-8.

4 Kamisawa T, Funata N, Hayashi Y, Eishi Y, Koike M, Tsuruta K, et al. A new clinicopathological entity of IgG4related autoimmune disease. J Gastroenterol. 2003;38(10):982-4.

5 Salvadori M, Tsalouchos A. Immunoglobulin G4-related kidney diseases: an updated review. World J Nephrol. 2018 Jan;7(1):29-40.

6 Quattrocchio G, Roccatello D. IgG4-related nephropathy. J Nephrol. 2016 Aug;29(4):487-93.

7 Kawano M, Saeki T. IgG4-related kidney disease-an update. Curr Opin Nephrol Hypertens. 2015 Mar;24(2):193-201.

8 Stone JH, Zen Y, Deshpande V. IgG4-related disease. N Engl J Med. 2012 Feb;366(6):539-51.

9 Hara S, Kawano M, Mizushima I, Yamada K, Fujita K, Harada K, et al. A condition closely mimicking IgG4related disease despite the absence of serum IgG4 elevation and IgG4-positive plasma cell infiltration. Mod Rheumatol. 2016 Sep;26(5):784-9.

10 Makiishi T, Shirase T, Hieda N, Maeda S. Immunoglobulin G4-related disease with scant tissue IgG4. BMJ Case Rep. 2013 Jun 6;2013. pii: bcr2013009800.

11 Khosroshahi A, Cheryk LA, Carruthers MN, Edwards JA, Bloch DB, Stone JH. Brief Report: spuriously low serum IgG4 concentrations caused by the prozone phenomenon in patients with IgG4-related disease. Arthritis Rheumatol. 2014 Jan;66(1):213-7.

12 Wang R, He D, Zhao L, Liang S, Liang D, Xu F, et al. Role of complement system in patients with biopsyproven immunoglobulin G4-related kidney disease. Hum Pathol. 2018;81:220-8. 


\section{Case Reports in Nephrology and Dialysis}

Case Rep Nephrol Dial 2019;9:49-54

DOI: $10.1159 / 000500296$

(c) 2019 www.karger.com/cnd

Wong et al.: IgG4-Related Kidney Disease: A Curious Case of Interstitial Nephritis with Hypocomplementemia
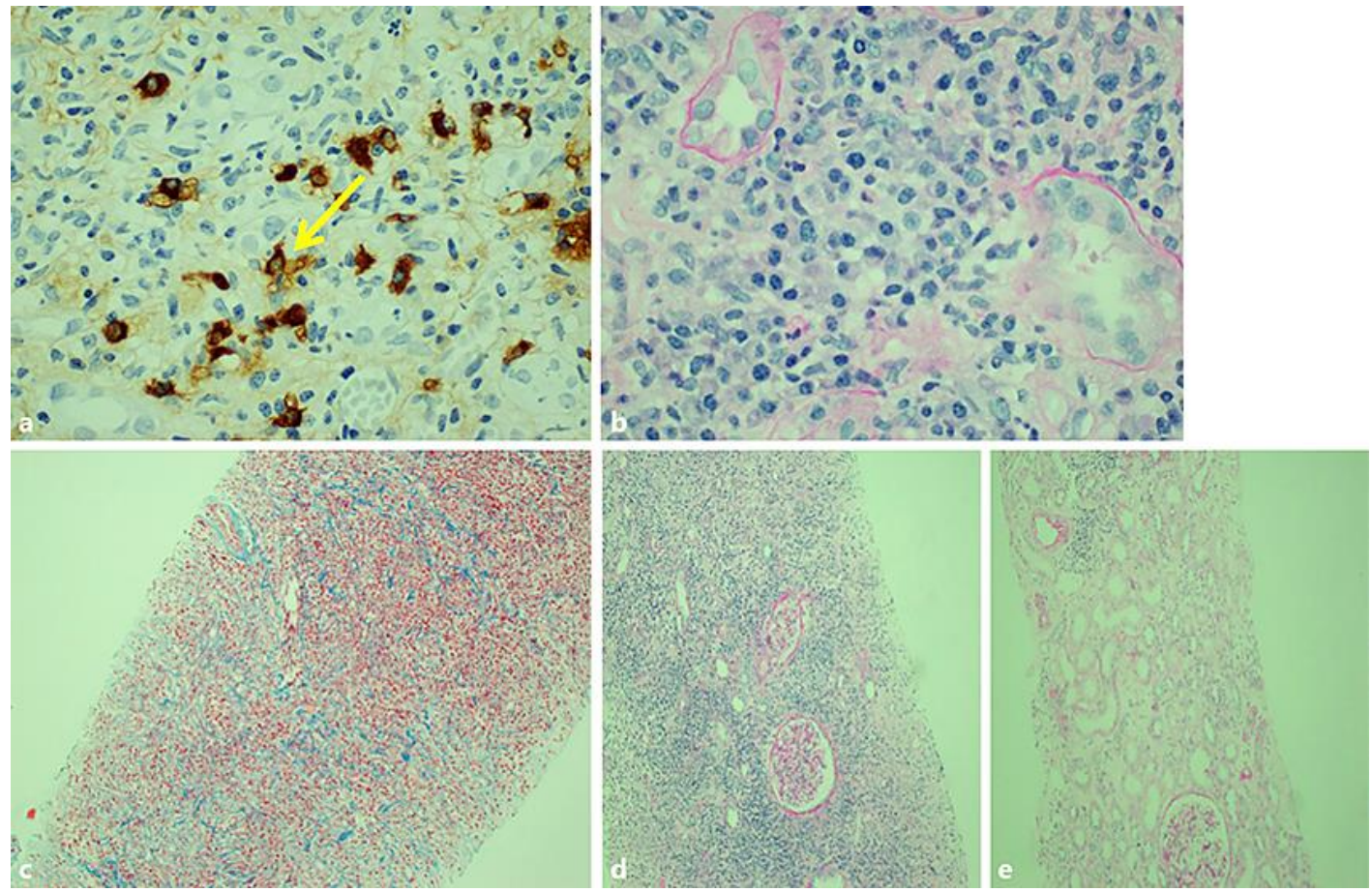

Fig. 1. a IgG4-positive plasma cells (arrow) on immunohistochemistry $(\times 400)$. b Extensive inflammation composed predominantly of mononuclear cells including prominent plasma cells admixed with scattered neutrophils and eosinophils (periodic acid-Schiff, $\times 400$ ). c Storiform pattern of interstitial fibrosis (Masson trichrome, $\times 100$ ). $\mathbf{d}$ Zonal character of tubulointerstitial inflammation with relative un-involvement of another zone $(\mathbf{e})$ (periodic acid-Schiff, $\times 100)$.

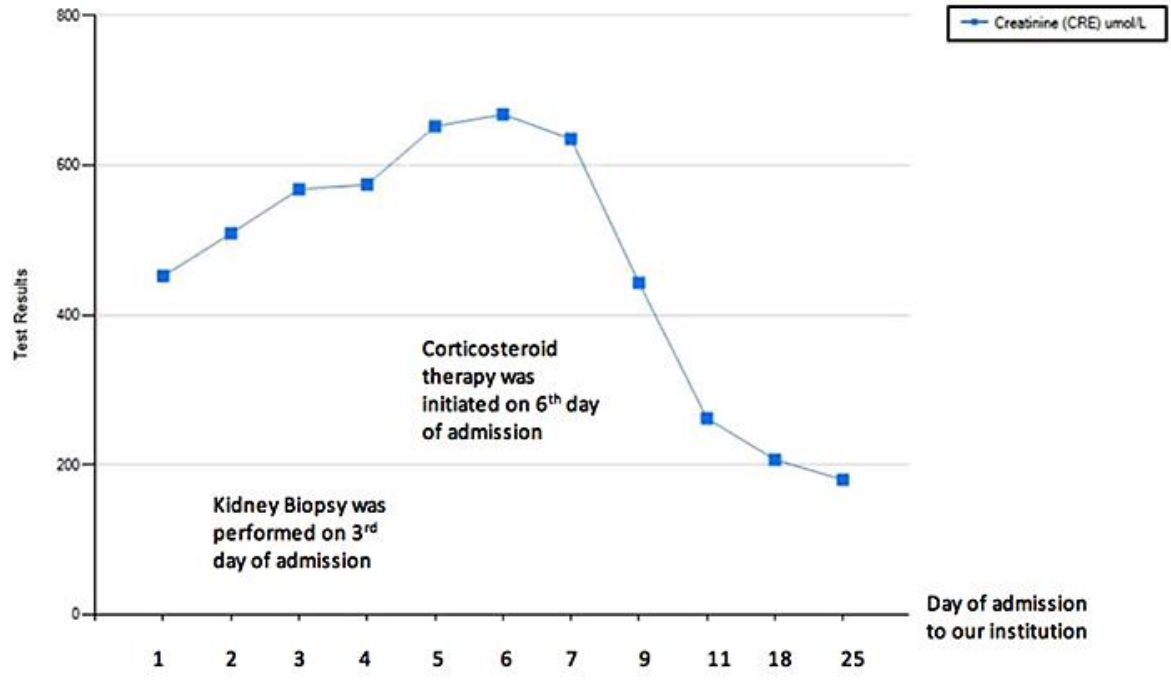

Fig. 2. Trend of creatinine. 